\title{
Uma abordagem sobre inteligência artificial e simulação, com uma aplicação na pecuária de corte nacional
}

\author{
Miguel Antonio Bueno da Costa \\ Professor Assistente DEP-UFSCar. \\ Doutorando em Engenharia de Sistemas na UNICAMP. \\ Departamento de Engenharia de Produção Universidade Federal de São Carlos-UFSCAR \\ Via Washington Luiz, km. 235, 13560 - São Carlos-SP
}

Palavras-chave: Inteligência Artificial, Simulação, Gado de Corte.

Key word: Artificial Intelligence, Simulation, Beef Cattle.

\section{RESUMO:}

UMA ABORDAGEM SOBRE INTELIGÊNCIA ARTIFICIAL E SIMULAÇÃO, COM UMA APLICAÇÃO NA PECUÁRIA DE CORTE NACIONAL: Este trabalho apresenta um histórico e uma visão geral sobre o potencial de a plicação de Inteligência Artificial (IA) em ambientes de Simulação. Como exemplo é apresentada uma aplicação dessa Nova Simulação (que utiliza conceitos de IA) na análise e síntese de sistemas de produção de gado de corte no Brasil.

\section{ABSTRACT:}

AN ARTIFICIAL INTELLIGENCE AND SIMULATION APPROACH WITH AN APPLICATION IN THE BRAZILIAN BEEF CATTLE PRODUCTION: This paper presents a general view on the potentialities of Artificial Intelligence ( $A I$ ) applications in Simulation environments. It illustrates through an example the use of this New Simulation (with AI concepts embodied) in the analysis and synthesis of brazilian beef cattle production.

Rec. 20/03/91 Rev. 16/05/91 Apr. 31/05/91 


\section{Introdução}

Inteligência Artificial, em particular Sistemas Especialistas (SE), são tópicos importantes na comunidade científica atual. Nos últimos anos têm ocorrido várias tentativas de aplicação de métodose "softwares" de IA no desenvolvimento de Simulações. Os sistemas assim gerados, utilizando-se da tecnologia deIA, permitem construções rápidas de modelos, aceleram as validações dos mesmos, e rodam com a base de conhecimento interna ao sistema/pacote. Este último fato permite que decisões sejam tomadas sem interromper o processo de simulação.

A IA tem suas raízes nos ensaios especulativos sobreo poder dos computadores, ralizados por Turing, em 1950 (Turing, 1950). Como coloca R.E.Shannon (1985), a IA como é conhecida hojcé resultado de um encontro de dez cientistas, interessados em computação simbólica, realizado em Dartmouth College, New Hampshire, em 1956. Entretanto, somente nos últimos anos a iA tornouse comercial, atuando em áreas bastante restritas.

Existem muitas definições para Inteligência Artificial,algumas filosóficas, outras mais pragmáticas, dependendo muito do campo de atuação dos autores das mesmas. Por exemplo:

Segundo E. Rich (17) "Inteligência Artificial é o estudo de como fazer computadores realizarem tarefas para as quais, até o momento, ohomem faz melhor". Uma definição simples mas que retrata com muita clareza o propósito da IA.

D. Waterman (1985) defineIA como "uma subárea da Ciência da Computaçãoqueobjetiva desenvolver programas computacionais inteligentes. Esses programas são: solucionadores de problemas, programas que me-
Ihoramsua própria performance, programas que interpretam linguagens, programasque reconhecem esquemas visuais, enfim que se comportamdemaneira queseria considerada inteligente se observada num homem".

Um ponto comum e intrínsico às definiçōes, aqui apresentadas ou não, é a necessidade de compreenssão dos processos humanos de aprendizagem, e a modelagem dos mesmos, permitindo a emulação desses processos numa máquina computacional.

Segundo R.Shannon (1985) a IA atua em dois grandes campos: i. Imitação das habilidades humanas (visão, fala, gestos, ...) c ii. Duplicaçãode resultados estabelecidos pelo homem através de sua habilidade e/ou experiência. Para exemplificar melhor a colocação acima, apresentaremos algumas áreas de pesquisa da IA :

- Solucionadores de Problemas (abordagem heurística que, sem analisar todas as alternativas, mostra um caminho com boa chance de êxito);

-Raciocínio Lógico (dedução);

- Processamento de Linguagem Natural (tradução e compreensão de textos);

- Robótica e Visão (manupulação de objetos, seqüenciamentode tarefas, reconhecimento de padrões);

- Programação Automática (geradores automáticos de programas computacionais);

- Aprendizagem (o sistema aprendendo com sua própria experiência, melhorando sua performance);

-Sistemas Especialistas (armazenamoconhecimento de uma área específica de atuação, utilizando-o como suporte à tomada de decisão). 
J.G. Moser (1986) coloca IA como um termoabrangentequeinclui diversasáreas, mas enfatiza que a área que está causando maior impacto nas ciências administrativas é, sem dúvida, Sistemas Especialistas (SE). Ele define SE, também chamado Sistema Baseado em Conhecimento, como um "software" capaz de acessar e processar logicamente as informações que uma pessoa, especialista numa área particular de conhecimento, utilizaria para tomar uma decisão.

Da mesma forma que para IA, são várias as definições para Sistemas Especialistas. Apresentaremos a seguir algumas:

M.S.Fox (1990): “É um programa computacional que limita o comportamento de exploração (busca) dos especialistas humanos na solução de problemas".

Kerchoff (1986): “Sãosistemas de processamento de informações que utilizam uma combinaçãode raciocínio simbólicocom processamento de dados".

Shannon (1985): São sistemas projetados para compilar a experiência de um certo número de especialistas, de um campo de atuação bem definido, numa série de regras. Essas regras serão utilizadas para gerar inferências e súgerir ao usuário um curso de ação no tratamento de um problema".

De uma forma geral as definições de Sistemas Especialistas possuem alguns pontos comuns:

- São sistemas computacionais (programas computacionais);

- Processam informações obtidas junto à especialistascomárea de atuaçãobem definida e restrita;

- Trabalham com regras e dados incertos;
-Utilizam raciocínio simbólico;

- Explicam a linha de raciocínio e justificam suas conclusões;

- Possuem interação amigável.

Dentro do exposto, uma definição que reflete bem o que entendemos por SE é dada por D.Waterman (1985): "Sistema Especialista é um programa computacional que utiliza conhecimentos deespecialistas (peritos) para alcançar altos níveis de performance numa área restrita doconhecimento. Esses programas representam conhecimento simbolicamente, examinam cexplicam seus processos de raciocínio, e tratam com problemas de áreas que requerem anos de treinamento especial e educação para que um homem possa dominá-lo".

Para atender ao que se propõe, um típico sistema especialista deve ser composto de:

- Uma Base de Dados Global;

- Uma Base de Conhecimento;

- Uma Estrutura de Interferências.

A Base de Dados Global é composta pelo conhecimentodeclarativo, contendoos fatos representativos da realidade do problema particular tratado. a Base de Conhecimento descreve os fatos e as heurísticas que representam o conhecimento do especialista (conhecimento relacional). A Estrutura (máquina) de Inferências define como os dadoseconhecimentos scrãomanipulados para se chegar à solução do problema.

Sistemas Especialistas diferem um pouco das pesquisas puras em IA, porque seu objetivoprincipal nãoéentender os mecanismos básicos utilizados peloespecialista para chegar a algum resultado, mas sim duplicarcon- 
sistentemente esses resultados. SE são projetados para compilar a experiência de especialistas de um campo restrito de trabalho, transformá-la $\mathrm{em}$ regras que permitam inferências, e sugerir cursos de ação ao usuário.

\section{A nova simulação}

Existe uma relação estreita entre simulaçãoe IA, devidoàs similaridades metodológicas. Ambas tentam modelar a realidade, representando os objetos e as relações entre eles. Segundo Doukidis (1987), nos anos60e 70, os mesmos conceitos de simulação foram utilizados no campo da IA, para solucionar problemas como configuração de computadores e diagnósticos médicos.

Para R.M.O'Keefe (1986) o que torna os métodos de Simulação e SE similares, é que eles são baseados em uma representação modular de um sistema, com um mecanismo de inferência que dirige essa representação.

K.J.Murray (1988) cita que muitos pesquisadoresenvolvidos com simulaçãoenxergam SE e Simulação como tecnologias complementares, que podem ser combinadas para fornccer um suporte poderoso para tomada de decisão. Tradicionalmente um projetista de sistemas de simulação constrói scu modelo utilizando-se de uma linguagem de programação geral (como FORTRAN e PASCAL por exemplo) ou de uma linguagem própria de simulação(GPSS, SIMULA, SIMSCRIPT, eoutras). O modelocntãoé validado, exccutadoe os resultados analisados cinterpretados. Várias partes desse ciclo de desenvolvimentodosistema podem serautomatizadas, utilizando-se ferramentas e técnicas de IA.

Com relaçãoà análise de resultados, muitos sistemas incluem a presença de uma tomador de decisão que tem con trole sobre o que acontece nosistema. Para simular tal sistema, o processo precisa ter acesso ao tomador de dicisãoquando necessário, ou este precisa ser modelado. A interpretação de resultados altamente complexos da simulação só pode ser feita por especialistas. A escassez co custodo serviçoespecializado para interpretar as saídas podem criar um sério gargalo e muitas vezesanular as vantagens da simulaçãocomo uma ferramenta de plancjamento gerencial.

Moser (1986) propõc a criaçãode sistemas integrados de suporte à decisão, desenvolvidos pela combinação da habilidade da simulação para prever valores de conjuntos complexos de variáveis, com a habilidadede raciocínio dos Sistemas Especialistas. Esses sistemas permitem análises das saídas da simulação, fazendo as inferências necessárias.

A combinação da simulação tradicional com os conceitos de IA (cm especial SE) gera uma poderosa ferramenta de suporte ao projeto, conhecida como Simulação Baseada cm Conhecimento (SBC) (Reddy, 1987). Essa abordagem tansforma a simulação, de uma ferramenta descritiva, numa ferramenta prescritiva.

A simulaçãopor nóschamada de tradicional (que não incorpora conceitos de IA) possui alguns pontos fracos, que inibem a sua maior utilizaçãoe sobrecarregam os projetistase programadores quando do desenvolvimento do modelo. Algumas desvantagens dessa simulação, que scrão comentadasa seguir, podem ser vistas como particularidades de uma afirmação mais ampla dada por Evans (1984), que diz: "Uma das maiores limitações da Simulação Tradicional é a sua inabilidade de modelar comportamentos inteligentes"

A fase de formulação de um modelo de simulação discreta é um procedimento custosoelongo, porque normalmente os programas auxiliados por computador não se preocupam com essa fase. As facilidades de 
Uma Abordagem Sobre Inteligência Artificial e Simulação, com Uma Aplicação na Pecuária de Corte Nacional

comunicaçãocntre usuáriose projetistas são, via de regra, inadequadas, nãoexistind o ferramentas que façam essa interação de forma mais amigável. Uma forma decontornaresse problema seria a utilização de geradores automáticos de programas desimulação(Balmer \& Paul, 1986).

A simulação tradicional, por trabalhar através de abordagens procedurais, amarra o sistema. As regras de decisão, uma vez codificadas, são fixas, tornando o sistema pouco flexível. Se essas regras pudessem ser construídasem separado da simulação, numa linguagem facilmente modificável, o resultado seria um sistema mais malcável. Isso facilitaria a manutenção dosistema, permitindo que novos conhecimentos fossem incorporados, sem afetar o módulo de controle existente.

Uma limitação bastante crítica da simulação tradicional é a não preocupação com a análise de resul tados c inferência ao final do processo de simulação. Normalmente o que ocorre é a apresentação de números, sem qualquer comentário mais prof undo. $\mathrm{O}$ modelode simulação fornece uma previsão. Ele prevê as conseqüências de um curso de ação escolhido entre muitos pertencentes a um cenário. A Simulação Bascada em Conhecimento fornece uma preścrição como resultado. Dada uma meta a ser atingida, a SBC sugere um curso de ação a ser seguido. esse procedimentoé de extrema importância uma vez que, como já foi comentado anteirormente, a análise de resultados complexos de simulação, é feita externamente ao "software", por especialistas caros e raros.

\section{Características da simulação baseada em conhecimento}

Podemos sintetizar o processo da simulação tradicional nos seguintes passos: a) Formulação do Problema, utilizando fluxogramas, formas estruturadas, diagramas ciclo-atividades, etc.;

b) Desenvolvimentodo Modelo. Pode ser utilizada uma linguagem de aplicação geral (FORTRAN, PASCAL, ALGOL, ...). ou uma linguagem específica de simulação (GPSS, SIMULA ,...); c) Verificação do Modelo. Examinar scasespecificações foram implementadas corretamente (se osistema foi construido corretamente);

d) Validação do Modelo. Verificar se a performance do sistema satisfaz as necessidades (se construiu o sistema certo);

e) Exccução do Modelo;

f) Análise de Resultados (decidir sobre novas rodadas de simulação).

A SBC temo propósitode automatizar, na emdida do possível, todas as a tividades "inteligentes" do processo de simulação, atualmente realizadas por especialistas. Nesse sentido propõe a utilização de sistemas especialistas para:

- Organizar e conservar conhecimentos;

- Permitir acesso a conhecimentos só disponiveis num especialista;

- Validar os processos de raciocínio do usuário, analisando os resultados da simulação;

- Evitar altas complexidades, utilizando raciocínio qualitativo em vez de métodos matemáticos.

Dentro do exposto, as atividades que caracterizam uma Simulação Baseada em Conhecimento, segundo Reddy (1987), são: 
a) Receber a descrição de um problema e sintetizar um modelo de simulação, consultando uma base de conehcimento apropriada;

b) Receber uma meta (em forma de um conjunto de expectativas ou restrições), selecionar um modelo, determinar a performance, gerar o espaço de pesquisa dos cenários possíveis, rodar e recomendar o cenário que satisfaz a meta cstabelecida;

c) Explicar a razão de certos cenários terem sido escolhidos c porque um cenário particular foi recomendado;

d) Aprender da experiência;

e) Apresentar o modelo resultante, com alto grau de tansparência para o usuário.

Devidoà utilização muitas vezes errônea do termo "Simulação Inteligente", Oren (1987a), (1987b) criou o termo "Simulação Cognizante" para diferenciar do que ele chama de Simulação Baseada em Conhecimento. Uma (cognitiva) que utilizando ferramentas de IA emularia habilidades humanas, como interpretação de textos, modelagem, análisc de resultados, análise das tomadas de decisões, etc. Outra ("cognizante") que se utilizaria das atividades cognitivas para aprender com o desenrolar do processo de simulação, ampliando sua base deconhecimento. Nesse últimocaso se inclui a Simulação Introspectiva onde o ambiente de simulação pode analisar trajetórias da própria simulação, agrupando conhecimentos do comporta mento do modeloe utilizando-os como conseqüências. Esses modelos introspectivos podem ser explorados para criar um modelo de SBC que aprenda, istoé, aumente o seu conhecimento com o aumento do número de simulações.

\section{Exemplo de aplicação na pecuária de corte nacional}

A origem desse trabalho se deve a um projeto apresentado pela Empresa Brasileira de Pesquisa Agropecuária - EMBRAPA/ UEPAE, São Carlos, SP, ao Conselho Nacional de Pesquisa de Gado de Corte-CNPGC/ Campo Grande, MS.

Os objetivos iniciais do trabalho estavam atrelados ao sistema americano TAMU, da Texas A \& MUniversity. Esse sistema, desenvolvido por J.O. Sanders e T.C. Cartwright (1977), (1979a), (1979b), nos anos 70, foi aperfeiçoadona década de 80 por J.F. Baker (1982), sendo responsável por inúmeras a plicações em diferentes regiōes do mundo. O TAMU foi programado em FORTRAN IV, para rodar numa máquina Amdahl 470V/6-II de grande porte, utilizando um compilador FORTRAN H estendido. O sistema se propunha a fazer (e faz) análises de sistemas de produção de gado de corte, utilizando conceitos de simulação.

Uma primeira tentativa foi a de adaptaro TAMU para ascondições brasileiras, alterando as funções associadas aos animais e ambiente, e fazê-lo rodar numa máquina IBM $370 / 148$ ou maior. Essa idéia não vingou, uma vez que não foi possivel ter acesso ao sistema americano.

Foi desenvolvido então um sistema, nos mesmos moldes do TAMU, voltado para atender o pequeno pecuarista nacional. Em decorrência desse novo objetivo, o sistema foi projetado para rodar em microcomputadores da linha IBM PC. Dessa forma não fica difícil a um pequeno pecuarista ter acesso às vantagens computacionais, fornecidas por essa ferramenta de a poio à decisão (no caso o modelo), a um baixo custo. 
* Característica Principais do Modelo

- Pode ser processado em qualquer microcomputador da linha IBM PC;

- Foi programado cm PASCAL;

- Utiliza um módulocentral de simulação, que mensalmente emula as condições de um rebanho de gado de corte, em relação às funções de Concepção, Nascimento, Crescimento Morte.

Todos os animais sãoclassificados deacordocom a sua idadce sexo. A pós cada rodada de simulação, cada animal é reclassificado, com scu vetor de dadosatualizado (peso, tamanhoestrutural, prenhez, cobertura, lactação, etc.);

- O modelo contém um módulo econômico que apresenta, a qualquer momento da simulação, a situação contábile financeira do sistema;

- Existe um módulo decisor inteligente que, durante a simulação, é responsável pelas decisões de compra e descarte de animais. Essas decisões, programadas a través de regras, ocorrem sem a necessidade de se interromper o processo de simulação. Esse módulo trabalha como um sistema conselheiro, incorporandooconhecimentodecspecialistas na área de compra e venda de animais.

Os módulos centrais do modelo (Simulaçãoe Decisor) atuam em paralelo (visto pelo usuário), ligados a dois bancos de dados (um contendo informações sobre os animais e ambiente, e outro com dados econômicos).

O módulo de simulação foi montado u tilizando-se um "shell" denominador ELSE (na época ainda em desenvolvimento), obtido a partir de notas de aula da London School of Economics and Political Science (Paul \& Balmer, 1985), (Chew et. al, 1985).
Esse módulo, recebendo informações dos animaise ambientee das comprasedescartes ocorridos, simula mensalmente a concepção, nascimento, crescimento e morte dos animais. Os resultados simulados são enviados de volta à base de dados dos animais, a tualizando-a.

As funções internasdo móduloSimulação são:

- Concepção : Estabelece a probabilidade de concepção a partir de informações sobre o início da ciclagem, grau de maturidade, condiçōesdoanimal, mudança no peso, lactação, intervalo pós parto e mês de monta;

-Nascimento: Controla o nascimento dos bezerros;

- Crescimento: Trata-se de uma função maior e mais delicada, que envolve todas as necessidades de manutençãa, prenhez, produção de leite, alteração de peso, etc. É utilizada por todos os animais mensalmente, independente de sua categoria;

- Morte: também utilizada por todos os animais, mensalmente, cujos parâmetros centrais são peso e idade.

O módulo decisor recebe as informações atual izadas pelos resultados đa simulaçãoocorrida, bem como as informações fornecidas pela base de dados econômicos. Feito sobre regras, que representam o conhecimento de especialistas na área de compra e venda de animais, csse módulo (analisando dados históricos c projeções futuras de preço de animais, e dados do rebanho) infere sobre decisões a serem tomadas em relação a compra e descartes. Os resultados são en tã đomunicados às bases de dados existentes, modificando-as.

Maiores informações sobre o modelo desenvolvido podem ser obtidas jun to ao autor desse trabalho. 


\section{Conclusões}

Segundo M.S.Fox (1990) “...Inteligência Artificial e Sistemas Especialistas não constituem panacéias, nem curiosidades científicas. Aocontrário, são tecnologias viáveisque permitem uma nova abordagem para solucionar muitos problemas de decisão". A combinação da metodologia da Simulação tradicional com conceitos de IA produz uma poderosa ferramenta de suporte ao projeto, conhecida como Simulação Baseada cm Conhecimento. Essa abordagem tansforma a simulação, de uma ferramenta descritiva, em uma ferramenta prescritiva.

A tendência de se ter cada vez mais sistemas de simulação expertos, e outras aplicações de IA em simulação, contribui para o avanço da mesma, tornando-a uma poderosa ferramenta de suporte à tomada de decisão em sistemas complexos.

\section{Bibliografia}

BAKER,J.F., "Evaluation of genotype-environment interactions in beef cattle production systems using a computer simulation model", Texas A\&M University, Texas, 1982.

BALMER,S.T.;PAUL,r.j., "CASM - The right environment for simulation", Journal of Operation Research Society, 37, pp. 443452, Great Britain, 1986.

CHEW,S.T.; PAUL,R.J.; BALMER,D.W., "Three-plase simulation modelling using an interective simulation program generator", London School of Economics and Political Science, London, 1985.

DOUKIDIS,G., "An anthology on the homology of simulation with artificial intelli- gence", Journal of operation Research Socicty, 38, 8, pp.701-712, Great Britain, 1987.

EVANS,J.B., "Simulation and intelligence", ,Hong Kong, 1984.

FOX,M.S., "AI and Expert Systems Myths, Legends and Facts AI Magazine, pp.8-20, Fev.1990.

KERCKOFFS,E.; VANSTEENKISTE,G.C., "The impact of advanced information processing on simulation-an illustrative revicw". Simulation, USA, 46, 1, pp.1726, Jan. 1986.

MOSER,J.G.,"Integration of artificial intelligence and simulation in a comprehensive decision suport system", Simulation, USA, 47, 6, pp.223-229, Dec. 1986.

MURRAY,K.J.; SHEPPARD,S.V., "Knowledge-based simulation model specification",Simulation, 50,3,pp.112-119,1988.

O'KEEFE,R.M., "Simulation and expert systems-a taxonomy and some examples", Simulation, 46, 1, pp.10-16, 1986.

ORENT,T.I., “Artificial Intelligenceand Simulation: from cognitive simulation toward cognizant simulation", Simulation, 48, 4, pp.129-130, 1987a.

ORENT,T.I.;ZEIGLER,B.P., "Artificial Intelligene in modelling and simulation: directions to explore", Simulation, 48, 4 . pp.131-134, 1987b.

PAUL,R.J.; BALMER,D., “Simulation Lecture Notes", London School of Economics and Political Science, Notas de Aula 1985.

PAUL,R.J.; CHEW,S.T., "Simulation modelling using an interactive simulation pro- 
gram generator", Journal of Operation Rescarch Socicty, 38,8, pp. 735-752, 1987.

REDDY,R., "Epistemology of Knowledge Based Simulation", Simulation, 48, 4, pp.162-166, 1987.

RICH,E., "Artificial Intelligence", McGrawHill, N.Y., 1983.

SANDERS,J.O., "Application of a becf cattle production model to the evalution of genetic sclection criteria", Texas A\&MUniversity, Texas, 1977.

SANDERS,J.O.; CARTWRIGHT,T., "A general cattle production systems model. I.
Description of the model.", Agricult.Systems, 4,3, pp.217, 1979a.

SANDERS,J.O.;CARTWRIGHT,T., "A general cattle production model. II. Simulation of animal performance.", Agricult. Systems, 4,4, pp.289, 1979 b.

SHANNON, R.E.; MAYER, R.; ADELSBERGER, H.H., "Expert systems and simulation", Simulation, 44,6,pp. 275-284, 1985.

TURING,A.M., "Computing machinery and intelligence", 49, pp.433-460), 1950.

WATERMAN, D.A., "A guide to ExpertSystems", Addison-Wesley 1985. 